\title{
Case Report on Labor and Delivery While Placenta Presence in Caesarian Vincent John*
}

Department of Gynaecology and Health Sciences, Faculty of Medicine, Universidade de Sao Paulo, Argentina

*Corresponding author: John V, Professor, Department of Gynaecology and Health Sciences, Faculty of Medicine, Universidade de Sao Paulo, Argentina, Tel:+55 11 3091-3116; E-mail: vincentjohnvj@rediffmail.com

Received date: June21, 2017; Accepted date: June28, 2017; Published date: July 03, 2017

Copyright: 2017 (c) John V. This is an open-access article distributed under the terms of the Creative Commons Attribution License, which permits unrestricted use, distribution, and reproduction in any medium, provided the original author and source are credited.

\begin{abstract}
Among the 65,000 live births in the Ukraine, 3 result in maternal demise. Some of these passings happen taking after difficulties related with placenta previa, which is show in roughly 1 in 200 pregnancies. Placenta previa happen when the placenta is situated in the lower some portion of the uterus, near or including the cervical opening. Commonly connected with or suspected in the nearness of placenta previa is the turmoil of placenta accreta, which is a type of unusual placentation and happens when the placenta imbeds defectively into the mass of the myometrium. The event rate of placenta accreta is around 1 in each 2,500 pregnancies. Placenta previa and placenta accreta are related with expanded maternal discharge and coming about expanded maternal mortality and morbidity. When the rate of cesarean conveyance expands, a relating increment in the event rate of placenta previa and placenta accreta has been watched. The rate of cesarean conveyance in the joined States is $31.1 \%$ and is at a record-breaking high. The expanded rate of cesarean conveyance is multifactorial. The number of essential cesarean conveyances being performed has expanded. In the interim, the quantity of vaginal births after cesarean conveyance has diminished. This diminished number has added to an expanded rate of rehashed cesarean conveyance. The reason for this case report is to survey the administration of a patient conceded for elective cesarean conveyance with known placenta previa and suspected placenta accreta. The case report will be trailed by a general talk identified with the administration of obstetric patients with placenta previa and placenta.
\end{abstract}

Keywords: Medical reports; Case studies; Pregnancy; Case reports; Placenta

\section{Introduction}

C-SECTION in the pregnancy is one of the main sources of mortality among ladies of tyke bearing age gathering. The greater part of these ectopic incubations are situated in the fallopian tube, ampulla being the most widely recognized area. Be that as it may, ectopic pregnancies are likewise known to happen in the cervix, ovary, past cesarean scar, and belly. Intramural pregnancy with implantation in a past cesarean area scar is most likely the rarest area for ectopic pregnancy [1].

This sort of pregnancy is inclined for entanglements like uterine burst, life-debilitating drain, and hypovolemic stun [2-4]. The genuine frequency of pregnancy happening in an uterine scar has not been resolved in light of the fact that so few cases have been accounted for in the writing. In any case, the occurrence of such cases is by all accounts on the ascent $[1,5]$. This may reflect both the expanding number of cesarean segments being performed and the more far reaching utilization of the transvaginal filter that permits prior location of such pregnancies.

\section{Case Report}

A 28-year-old lady had wanted routine antenatal examination following 2 months of amenorrhea and positive pee pregnancy test. She was generally asymptomatic. She had history of 2 past cesarean segments; initial one performed 5 years back for intrapartum fetal trouble and the second one preformed 2 years back because of finish placenta previa. She was exhorted routine first trimester sonography.
Transabdominal sonography supplemented by transvaginal sonography uncovered purge uterine cavity and discharge cervical channel with a gestational sac in front myometrium of lower uterine section (Figures 1-3).

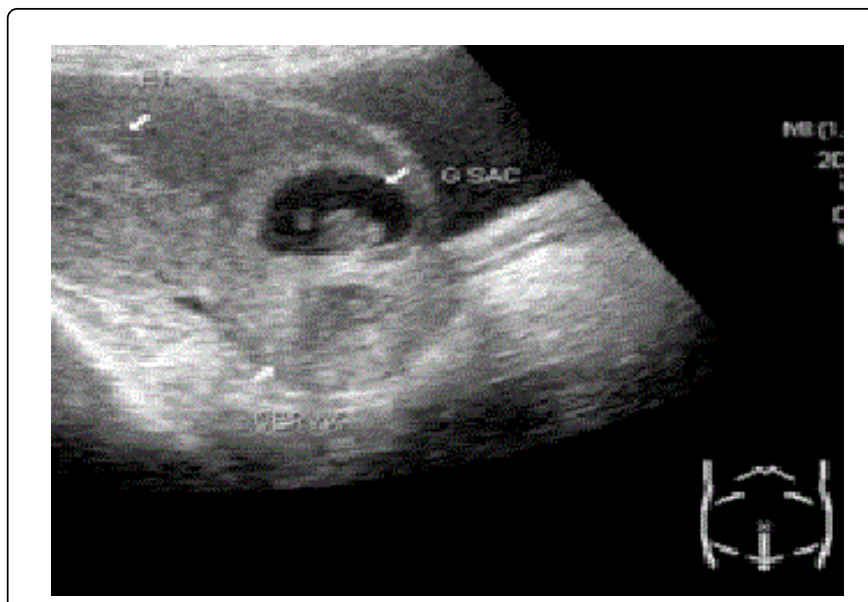

Figure 1: Transabdominal sonography showing empty uterine cavity and empty cervical canal with a gestational sac in anterior myometrium of lower uterine segment. The gestational sac shows a fetal pole within. Anterior myometrium anterior to the gestational sac is thinned out. 
Citation: John V (2017) Case Report on Labor and Delivery While Placenta Presence in Caesarian. Med Rep Case Stud 2: 139. doi: $10.4172 / 2572-5130.1000139$

Page 2 of 3



Figure 2: Transabdominal sonography showing gestational sac in anterior myometrium of lower uterine segment, which shows a fetal pole (thick white arrow) within.



Figure 3: Color Doppler examination showing that hyperechoic rim of choriodecidual reaction (thick white arrow) and umbilical cord (thin white arrow) show vascularity.

The gestational sac had a fetal shaft and yolk sac inside, demonstrating fetal cardiovascular action (Figure 4) and having normal gestational age of two months 1 day. Foremost myometrium front to the gestational sac was dispersed.

On Doppler examination, hyperechoic edge of choriodecidual response and umbilical string demonstrates vascularity. Transabdominal sonography indicating unfilled uterine pit and purge cervical channel.

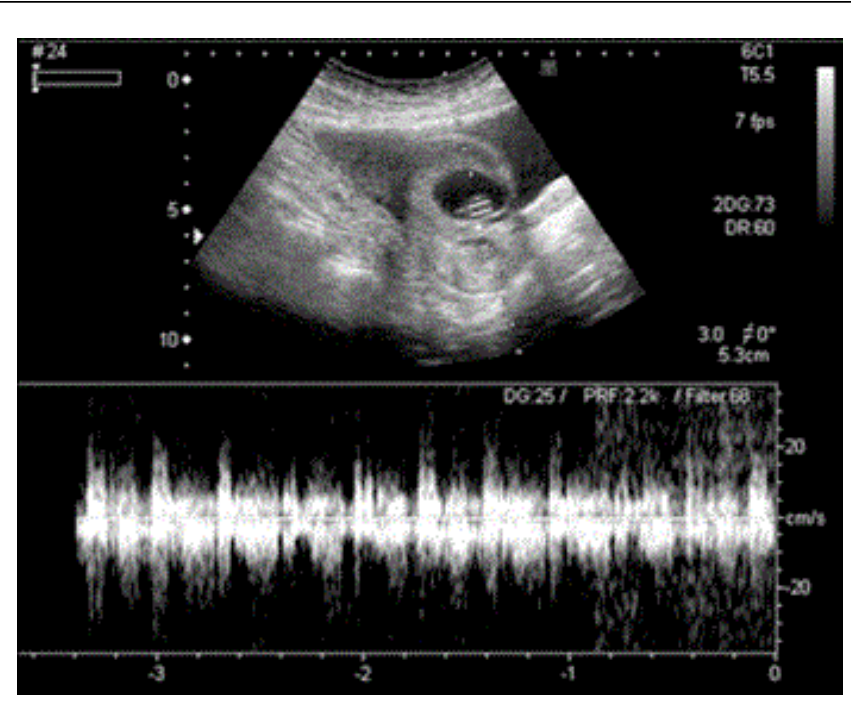

Figure 4: Spectral Doppler examination of the fetal pole demonstrating the presence of fetal cardiac activity.

\section{Discussion}

There are numerous speculations which clarify the event of intramural ectopic pregnancy. The most acknowledged hypothesis is by all accounts that the blastocyst attacks into the myometrium through an infinitesimal dehiscent tract, which might be the consequence of injury of a past cesarean segment or whatever other uterine surgery [6] or even after manual evacuation of the placenta [3]. Another system for intramural implantation might be in vitro preparation and incipient organism exchange, even without any past uterine surgery [7]. A cesarean segment is related with a future hazard for placental pathologies (e.g. placenta previa, placental suddenness, and placenta acccreta) and ectopic pregnancies. Nonetheless, a cesarean scar pregnancy is thought to be considerably more forceful than placenta previa or accreta in light of the fact that it attacks the myometrium in the primary trimester. Patients who have experienced different cesarean segments give off an impression of being at expanded hazard for in-scar implantation of the ensuing pregnancy on account of expanded scar surface zone $[4,8,9]$. With the coming of transvaginal sonography and with the utilization of saline imbuement, it is conceivable to survey postcaesarean area uterine divider respectability even in the nonpregnant state [4,10-12]. Cesarean segment scar deformity is distinguished by the nearness of liquid inside the entry point site [12] or any filling imperfection, which is characterized as a triangular anechoic structure at the assumed site of the scar.

\section{Conclusion}

Intramural pregnancy with implantation in a past cesarean area scar is likely the rarest area for ectopic pregnancy. This sort of pregnancy may end up plainly confused with uterine crack and life-undermining discharge. In this way, early conclusion of cesarean scar ectopic incubation utilizing sonography consolidated with Doppler stream imaging is of fundamental significance, trailed by affirmation of pelvic MRI if and when demonstrated. Albeit hopeful administration has been endeavored now and again, presently accessible information bolster end of such a pregnancy once the right determination is made. 
Citation: John V (2017) Case Report on Labor and Delivery While Placenta Presence in Caesarian. Med Rep Case Stud 2: 139. doi: $10.4172 / 2572-5130.1000139$

Page 3 of 3

\section{References}

1. Fylstra DL, Pound-Chang T, Miller MG, Cooper A, Miller KM (2002) Ectopic pregnancy within a Caesarian delivery scar: a case report. Am J Obstet Gyneco 187: 302-304.

2. Herman A, Weinraub Z, Avrech O, Maymon R, Ron-ElR, et al. (1995) Follow up and outcome of isthmic pregnancy located in a previous caesarean section scar. Br J Obstet Gynecol 102: 839-841.

3. Fylstra DL (2002) Ectopic pregnancy within a Caesarian scar: a review. Obstet Gynecol Surv 57: 537-543.

4. Jurkovic D, Hillaby K, Woelfer B, Lawrence A, Salim R, et al. (2003) Firsttrimester diagnosis and management of pregnancies implanted into the lower uterine segment Caesarian section scar. Ultrasound Obstet Gynecol 21: 220-227.

5. Ghezzi F, Lagana D, Franchi M, Fugazzola C, Bolis P (2002) Conservative treatment by chemotherapy and uterine arteries embolization of a Caesarian scar pregnancy. Eur J Obstet Gynecol Reprod Biol 103: 88-91.

6. Cheng PJ, Chueh HY, Soong YK (2003) Sonographic diagnosis of a uterine defect in a pregnancy at 6 weeks gestation with a history of curettage. Ultrasound Obstet Gynecol 21: 501-503.
7. Hamilton CJ, Legarth J, Jaroudi KA (1992) Intramural pregnancy after in vitro fertilization and embryo transfer. J Fertil Steril 57: 215-217.

8. Vial Y, Petignat P, Hohlfeld P (2000) Pregnancy in a Caesarian scar. Ultrasound Obstet Gynecol 16: 592-593.

9. Maymon R, Halperin R, Mendlovic S, Schneider D, Vaknin Z, et al. (2004) Ectopic pregnancies in Caesarean section scars: the 8 year experience of one medical centre. J Hum Reprod 19: 278-284.

10. Nawroth F, Foth D, Wilhelm L, Schmidt T, Warm M, et al. (2001) Conservative treatment of ectopic pregnancy in a Caesarian section scar with methotrexate: a case report. Eur J Obstet Gynecol 99: 135-137.

11. Sugawara J, Senoo M, Chisaka H, Yaegashi N, Okamura K (2005) Successful conservative treatment of a cesarean scar pregnancy with uterine artery embolization. Tohoku J Exp Med 206: 261-265.

12. Armstrong V, Hansen WF, Van Voorhis BJ, Syrop CH (2003) Detection of Caesarian scars by transvaginal ultrasound. J Obstet Gynecol 101: 61-65. 